\title{
Implementation of the method of adaptive management of electric power transmission in distribution electric networks of railways
}

\author{
Vasily Cheremisin ${ }^{1}$, and Evgeny Tretyakov ${ }^{1, *}$ \\ ${ }^{1}$ Omsk State Transport University, Karl Marx Ave., 35, Omsk, 644046, Russia
}

\begin{abstract}
The separation of the electric grid cluster rendering services related to transmission of electric power through electric grids from the railways electric power supply system within the Russian Railways holding will make it possible to proceed with to the modernization of the distribution electric networks of the railways at the expense of the investment component of the tariff. The innovative development of the grid company of the Russian Railways holding company is to be synchronized with its technological indicators with PJSC FGC UES and PJSC Rosseti and is aimed at creating a stable, efficient, flexible and intelligent electric power infrastructure. The aim of the present research is to increase the efficiency of electric power transmission in the distribution electric networks of railways due to the adaptive control of the regulators of the operation mode parameters. The implementation of the method of adaptive control of electric power transmission in distribution electric networks of railways based on the agent approach is presented.
\end{abstract}

\section{Introduction}

The issues related to the use of modern technologies in the electric networks of railway transport is reflected in the regulatory documents that determine the prospects for the development of the industry, for example, in the energy strategy of the Russian Railways for the period up to 2015 and for the future up to 2030 [1]. The strategy states that the railway transport is expected to provide the development of its own generation of electricity for nontraction needs. This, in turn, will require the creation of a system for managing electricity transmission based on the modern methods and means of controlling the parameters of the operation.

The main directions of the development of the power grid complex in Russia are presented in the strategy of the development of the electric grid complex of the Russian Federation, developed for the period until 2030 [2].

The basic requirements to perspective methods of quality management, transport and distribution of electric energy in the power supply system of stationary railway users: new two-way communication control systems; identification of the numerical values of

\footnotetext{
${ }^{*}$ Corresponding author: eugentr@mail.ru
} 
perturbation parameters necessary for making control-related decisions and modeling within the control systems.

\section{Objective formulation}

In energy systems, the most relevant and well-developed issues are the ones related to the optimization of modes of electrical networks for voltage and reactive power, and in some cases - for active power and frequency [3]. Until recently, it has been believed that local systems of automatic control of power supply facilities that use information within the facility, cannot provide the best operation of the energy system as a whole. One of the disadvantages of centralized control (optimization) is the need to collect, transmit and process in one place a significant amount of information on the status of the mode and the elements of the power system. The said control methods, as a rule, are implemented in high voltage electrical networks. A number of authors [4] develop a situational control method with the theory of fuzzy sets, which has a rough averaging of the mode parameters due to a finite number of control scenarios.

The most promising approach is the control of the electric power system based on the agent approach. Despite the fact that centralization in control is more profitable, with the increase in information flows it is significantly hampered. The agent approach assumes the integration of centralized and decentralized control [5-14]. For example, JSC FGC UES is implementing a pilot project to develop and implement an intelligent energy system with an actively adaptive network. As is known, PJSC FGC UES has mainly high-voltage transmission and distribution networks characterized by high observability. The main distinguishing feature of the agent approach is the set of control centers (parallelization of information flows). The implementation of the methods for the adaptive control of electricity transmission in the distribution networks of medium and low voltage railways based on the agent approach is currently not sufficiently developed in the scientific environment. In particular, the actual objective is to determine the control actions on local regulators to achieve control objectives.

\section{Theory}

Electrical distribution networks of railways are complex, time-varying objects in terms of topology and mode parameters. The main parameters of such an electrical network that can be changed (regulated) are: active and reactive power sources in the nodes, loads and current transmission lines, voltage in the nodes of the electrical network, transformer transformation ratios, etc.

Controlled objects can be the following devices

- transformers OLTC;

- transverse compensating devices (smoothly and stepwise adjustable);

- linear regulators and booster transformers;

- FACTS devices;

- sources of distributed generation;

- energy storage units;

- switching devices, etc.

The method for managing the transmission of electricity in railway distribution networks contains a distributed control architecture (controllers) based on measurements, ontologies and high data analysis speed, based on agent systems; electric sensors (current, voltage, power, power quality indicators, etc.) and sensors of non-electrical quantities (position of 
switching devices, tap-changers, compensating devices, etc.); local controllers of linear regulators [15-18].

All measuring devices and controllers are united according to their protocols into a single information field of the virtual network model within the boundaries of the power district (with the possibility of data exchange and transfer of control rights to neighboring power districts). Local controllers on the basis of their own computing facilities in real time solve the problem of controlling executive devices in a given power district with a frequency that is an order of magnitude higher than the speed of calculating the global control criterion for the whole section of the electric network in question. If the local regulator of the mode parameters fails, the system redistributes its functionality to neighboring ones, thus increasing the reliability of the power supply system.

Consider the section of the $35 \mathrm{kV}$ distribution network of the power supply system of the third-party railway users (Fig. 1) in the normal and post-emergency mode (switching off the transformer $1 \mathrm{~T}$ at the substation A-110).

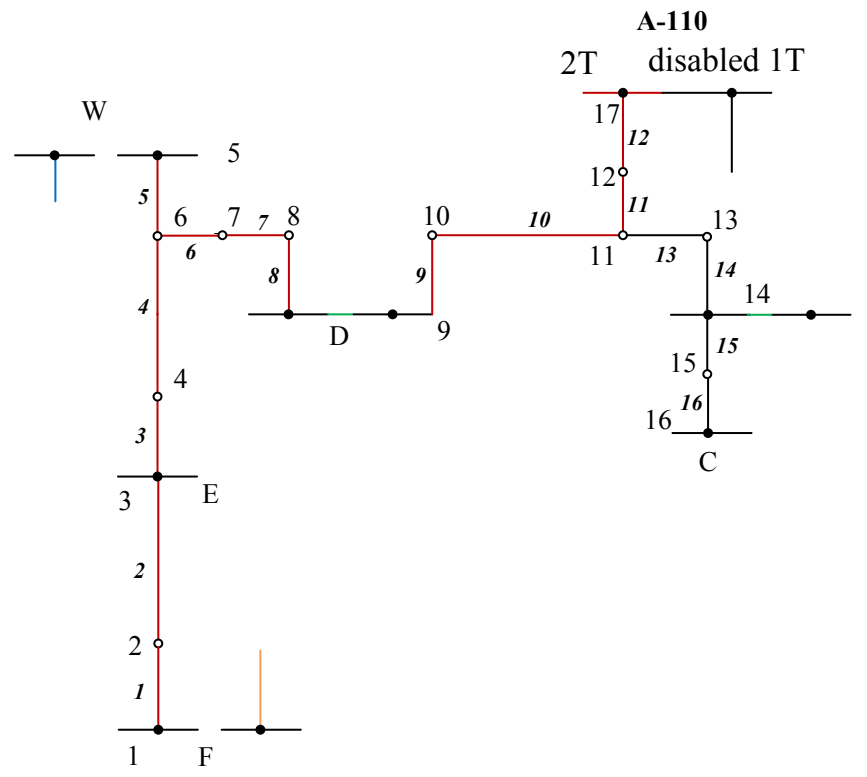

Fig. 1. Nodalization diagram.

With regard to the implementation of the method of adaptive control of electricity transmission in the distribution networks of railways in this article, we confine ourselves to the task of controlling the voltage in the nodes of the electrical network.

For steady-state modes, sensory and coherent elements of the electric network are detected through the sensor analysis of the corresponding Jacobi matrices (the matrix of partial derivatives of the secondary parameters of the mode in the case of a description in the form of a power balance) in the polar coordinate system [3].

The equations of steady-state modes in trigonometric form (in polar coordinates) have the following form: 


$$
\left\{\begin{array}{l}
U_{i} \sum_{j=0}^{n-1} Y_{i j} U_{j} \cos \left(\delta_{i}-\delta_{j}-\psi_{i j}\right)=P_{i} \\
U_{i} \sum_{j=0}^{n-1} Y_{i j} U_{j} \sin \left(\delta_{i}-\delta_{j}-\psi_{i j}\right)=Q_{i} .
\end{array}\right.
$$

Where $U_{i}, U_{j}$ are the nodal voltages; $Y_{i j}$ - components of nodal conduction matrix; $P_{i}, Q_{i}$ - active and reactive power in the node (generation minus the load).

The Jacobi matrix for power balance in polar coordinates:

$$
J=\left(\begin{array}{ll}
\frac{\partial P}{\partial \delta} & \frac{\partial P}{\partial U} \\
\frac{\partial Q}{\partial \delta} & \frac{\partial Q}{\partial U}
\end{array}\right) .
$$

The partial derivatives of unbalances (1) with respect to independent parameters (on the example of active power) take the following form:

$$
\begin{aligned}
& \frac{\partial P_{i}}{\partial \delta_{i}}=\sum_{j=1}^{n} U_{i} U_{j}\left[b_{i j} \cos \left(\delta_{i}-\delta_{j}\right)-g_{i j} \sin \left(\delta_{i}-\delta_{j}\right)\right] ; \\
& \frac{\partial P_{i}}{\partial \delta_{j}}=-U_{i} U_{j}\left[b_{i j} \cos \left(\delta_{i}-\delta_{j}\right)-g_{i j} \sin \left(\delta_{i}-\delta_{j}\right)\right] ; \\
& \frac{\partial P_{i}}{\partial U_{i}}=2 U_{i} g_{i j}+\sum_{j=1}^{n} U_{j}\left[g_{i j} \cos \left(\delta_{i}-\delta_{j}\right)+b_{i j} \sin \left(\delta_{i}-\delta_{j}\right)\right] ; \\
& \frac{\partial P_{i}}{\partial U j}=U_{i}\left[g_{i j} \cos \left(\delta_{i}-\delta_{j}\right)+b_{i j} \sin \left(\delta_{i}-\delta_{j}\right)\right] .
\end{aligned}
$$

As a result of the sensory analysis of the section of the electric network in question, the most sensory nodes are: by reactive power - 3, 5. The "weakest" branches by voltage and power losses: 10 (length $12.4 \mathrm{~km}$, wire AC 95), $3+4$ (length $1.7+13.7 \mathrm{~km}$, AC 70 wire), 7 (length of $8.1 \mathrm{~km}$, AC-95 wire).

The dependence of the losses of active power in the network on the loads of individual nodes is close to quadratic, the relative increments of losses and power - to linear. The target management function has the following form:

$$
F=\sum_{i=1}^{n}\left[\sum_{j=1}^{m_{i}}\left(k_{P} \Delta P_{j}+k_{Q} \Delta Q_{j}\right)\right]+k_{L} \Delta L \rightarrow \min ,\left\{\begin{array}{l}
\sum_{i=1}^{n}\left[\sum_{j=1}^{m_{i}}\left(a_{j k} \Delta P_{j}+b_{j k} \Delta Q_{j}\right)\right]+c_{L} \Delta L=\Delta U_{k} ; \\
L^{\min } \leq L^{0}+\Delta L \leq L^{\max } \\
P_{j}^{\min } \leq P_{j}^{0}+\Delta P_{j} \leq P_{j}^{\max } \\
Q_{j}^{\min } \leq Q_{j}^{0}+\Delta Q_{j} \leq Q_{j}^{\max } \\
\forall j=1, \ldots, m_{i .}
\end{array}\right.
$$

$\mathrm{n}$-the number of sections connected to the same bus with a transformer; $L^{0}, L^{\min }, L^{\max }$ adjustment of the regulator (branches) of the transformer with the on-load tap-changer and its limitation (in p.u.); $k_{P}, k_{Q}$ - weighing factors for active and reactive power ("cost" of 
regulation); $k_{L}$ - weighting factor for controlling the actions of the on-load tap-changer, characterizing the sensitivity of the voltage on the bus k TP when the step of the on-load tapchanger of the transformer changes to high voltage / medium voltage; $P_{j}, Q_{j}$, active and reactive power (generation and (or) consumption); $a_{j k}, b_{j k}$ - voltage sensitivity coefficients for active and reactive power.

Thus, it follows from (4) that

$$
\left(\frac{k_{P}}{a_{j k}} \Delta P_{j}+\frac{k_{Q}}{b_{j k}} \Delta Q_{j}\right)=\Delta U_{k} .
$$

To determine the control actions, in the form of a change in the active $\Delta P$ and reactive $\Delta Q$ increments, the following linearized equation is used:

$$
\left(\begin{array}{c}
\Delta \delta \\
\Delta U
\end{array}\right)=J^{-1}\left(\begin{array}{c}
\Delta P \\
\Delta Q
\end{array}\right)
$$

Where $\Delta \delta, \Delta U$ are changes in phases and voltage modules; $J$ - the Jacobi matrix $J=\left(\begin{array}{ll}J_{P \delta} & J_{P U} \\ J_{Q \delta} & J_{Q U}\end{array}\right)$.

The values of control actions on active and reactive power on the nodes of the electrical network (from the expression (6)):

$$
\begin{aligned}
& \Delta P=\left(J_{P U}-J_{P \delta} J_{Q \delta}^{-1} J_{Q U}\right) \Delta U \\
& \Delta Q=\left(J_{Q U}-J_{Q \delta} J_{P \delta}^{-1} J_{Q U}\right) \Delta U
\end{aligned}
$$

The effectiveness of control actions is determined from the relations $\left(k_{Q} / b_{i k}\right)$ and $\left(k_{P} / a_{j k}\right)$

\section{The Results of the experiments}

As a result of the ranking in descending order of the ratios $\left(k_{Q} / b_{i k}\right)$ and $\left(k_{P} / a_{j k}\right)$ the most effective reactive power sources at nodes 3 and 5 were iteratively determined to achieve objective 1 (Fig. 2).

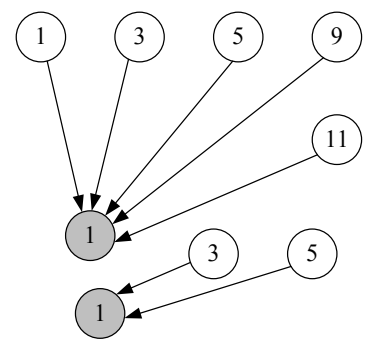

Fig. 2. Distribution of local regulators for voltage regulation as a result of ranking.

To stabilize the voltage ( $\Delta U_{k} \rightarrow \min$ ) due to the operative control of local regulators at a particular time, the optimal control actions for the installed linear regulators - reactive power sources at nodes 3 and 5 of the specified devices - were determined by expressions (4) - (7) (Fig.3). 

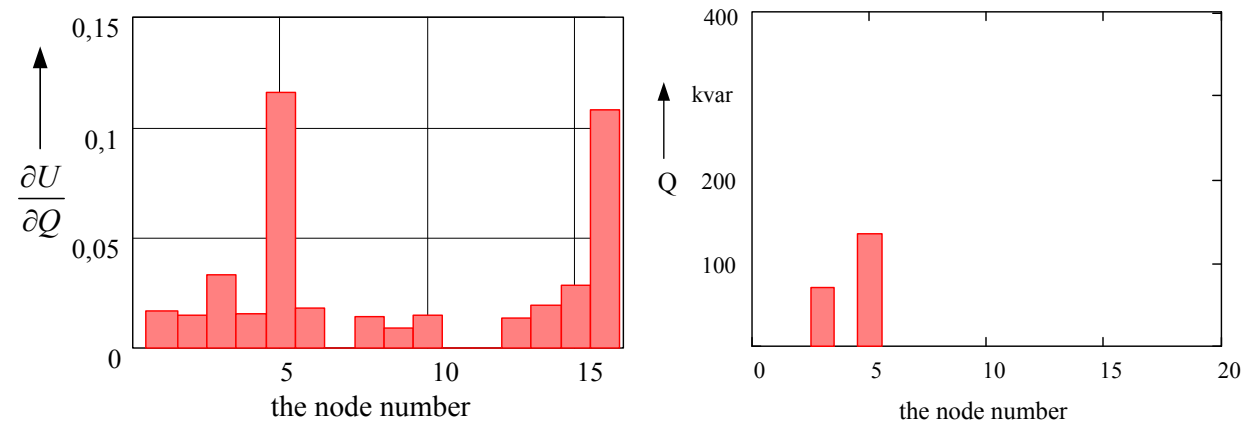

Fig. 3. The sensitivity of the voltage to the change in reactive power and the control effects of reactive power sources.

Table shows the results of modeling of the normal and post-emergency modes of the electrical network with the calculated regulators settings. Fig. 4 shows the reduction in voltage drops and power losses in the lines after the injection of reactive power in nodes 3 and 5 .
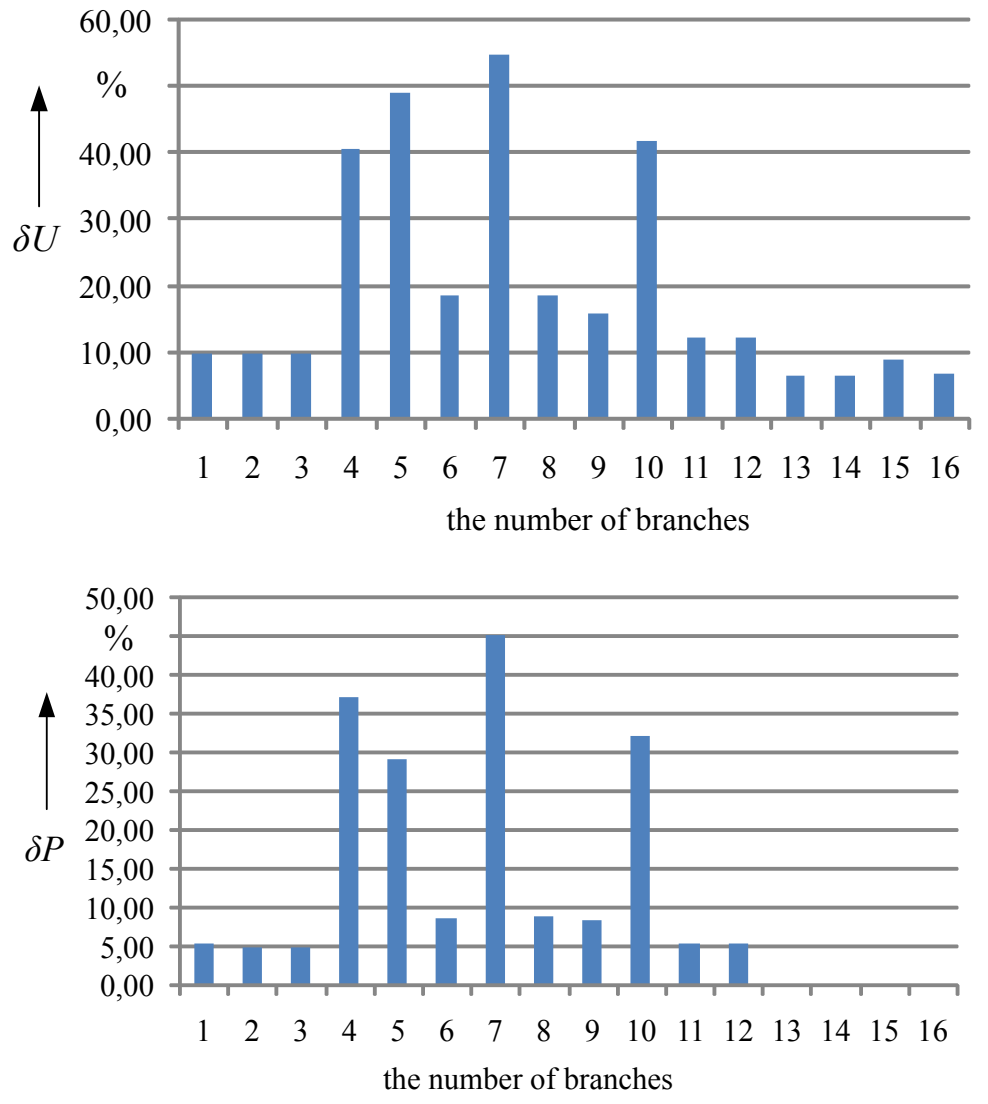

Fig. 4. Reduction of voltage drops and power losses in the lines. 
Table 1. Results of modeling of normal and post-emergency modes.

\begin{tabular}{|l|l|l|l|l|}
\hline \multirow{3}{*}{ Node } & \multicolumn{3}{|c|}{ Multi-agent Control } & \multicolumn{2}{c|}{ Centralized Control } \\
\cline { 2 - 5 } & \multicolumn{4}{|c|}{ Voltage, $\mathrm{kV}$} \\
\cline { 2 - 5 } & Normal & Post-emergency & Normal & Post-emergency \\
\hline 1 & 35.11 & 35.09 & 34.51 & 33.21 \\
\hline 2 & 35.31 & 35.13 & 34.54 & 33.27 \\
\hline 3 & 35.47 & 35.39 & 34.74 & 33.80 \\
\hline 4 & 35.72 & 35.49 & 34.80 & 33.86 \\
\hline 5 & 36.24 & 35.94 & 35.36 & 34.23 \\
\hline 6 & 35.86 & 36.05 & 34.91 & 34.10 \\
\hline 7 & 36.92 & 36.30 & 35.64 & 34,12 \\
\hline 8 & 36.82 & 36.76 & 36.18 & 35.60 \\
\hline 9 & 36.96 & 36.87 & 36.30 & 35.80 \\
\hline 10 & 37.04 & 37.02 & 36.47 & 35.90 \\
\hline 11 & 37.61 & 37.98 & 37.56 & 36.78 \\
\hline 12 & 37.69 & 38.18 & 37.68 & 37.0 \\
\hline 13 & 37.80 & 37.78 & 37.80 & 37.80 \\
\hline 14 & - & 37.43 & - & 37.65 \\
\hline 15 & - & 37.43 & - & 37.64 \\
\hline 16 & - & 37.42 & - & 37.20 \\
\hline
\end{tabular}

A distinctive feature of the agent approach to the electricity transmission control in distribution electrical networks is that the control actions on local controllers of reactive power sources were calculated not on the basis of cumbersome optimization of the mode parameters of the entire network, but were based on the choice of regulators by ranking them in terms of the auction type and optimal settings determination.

\section{Discussion of the results}

As follows from the data of Table. 1, the coordinated multi-agent control is better able to cope with the task of stabilizing the voltage in the nodes of the electrical network. Modern compensating devices are equipped with local controllers capable of providing a specified level of voltage at the point of connection to the electrical network.

Autonomous operation of reactive power sources in automatic mode stabilizes the voltage at the connection point, which does not always correspond to the purposes of voltage control in neighboring nodes of the power grid. The use of coordinated control of several of these devices allows maintaining the required voltage level taking into account power injections in the adjacent areas of the power grid.

The results of simulation modeling show an average $25 \%$ decrease in voltage drops, $15 \%$ drop in power losses in the lines using the specified settings of linear regulators on the basis of the agent approach.

\section{Summary and conclusion}

Thus, the results of the studies have shown the effectiveness of the method of controlling the transport of electricity in the distribution electric networks of railways due to the adaptive control of reactive power regulators. The use of the coordinated voltage regulation by local regulators, including the on-load tap-changer in the power center, allows not only to improve the voltage quality, but also to reduce the power losses in the network. 


\section{References}

1. Strategy of development of the power grid complex of the Russian Federation (for the period until 2030) Approved by the order of the Government of the Russian Federation N 511-p. (2013)

2. Energy strategy of the holding «Russian Railways» for the period until 2015 and for the future up to 2030, JSC Russian Railways (2011)

3. A. Augugliaro, L. Dusonchet, S. Favazza, E. Riva, IEEE Trans. on Power Delivery 193, 1516 - 1527 (2004)

4. D. Morales, R. Lopez, IEEE Trans. on Power Systems 11, 15 - 16 (2007)

5. X. Han, A.M. Kosek, D.E.M. Bondy, H.W. Bindner, Y. Shi, D.V. Tackie, J. Mehmedalic, F. Thordarson, Intelligent Energy Systems (IWIES) 2014 IEEE International Workshop on, 46 - 51 (2014)

6. H.B. Wu, C.Y. Huang, M. Ding, B. Zhao, P. Li, Journal of Modern Power Systems and Clean Energy 5(5), 504 - 511 (2017)

7. X. Ye, J. Le, Y. Liu, W. Zhou, K. Liu, Journal of Modern Power Systems and Clean Energy 6(1), 85 - 94 (2018)

8. E. Ghiani, F. Pilo, Journal of Modern Power Systems and Clean Energy 3(4), $504-511$ (2015)

9. M. Juamperez, G.Y. Yang, S.B. Kjaer, Journal of Modern Power Systems and Clean Energy 4(2), 319 - 328 (2014)

10. A. Morattab, O. Akhrif, M. Saad, Iet Generation Transmissin\&Distribunion 11-18, 4546 -4555 (2017)

11. C. Dou, D. Zhang, D. Yue, Y. Zheng, IEEE Access 5, 11381 - 11390 (2017)

12. K. Mohd, S. Mohd, Z. Mingye, K. Sheikh, A. Sheikh, H. Faten, S. Nira, T. Muhamad, H. Azmi, G. Qinglai, Innovative Smart Grid Technologies Asia (ISGT Asia) IEEE, 769 -773 (2014)

13. Y. Xu, Z. Dong, R. Zhang, D. Hill, IEEE transactions on power systems 32-6, 4398 4408 (2016)

14. H. Farag, E. El-Saadany, L. Chaar, Proceedings of the 2011 international conference on innovations in information technology (IIT'11) (Abu Dhabi, 2011)

15. H. Mehrjerdi, S. Lefebvre, M. Saad, D. Asber, H. Mehrjerdi, S. Lefebvre, M. Saad, D. Asber, Eliminating voltage violations in power systems using secondary voltage control and decentralized neural network, Proc. IEEE PES General Meeting (Vancouver, 2013)

16. W. Hu, L. Zheng, Q. Lu, Y. Min, Energies 6, 2949 - 2965 (2013)

17. T. Niknam, M. Zare, J. Aghaei, IEEE Trans. Power Del. 27(4), 2004 - 2019 (2012)

18. H.E. Farag, E.F. El-Saadany, R.A. Seethapathy, IEEE Trans Smart Grid 3(1), 271-281 (2012) 